\title{
New data about Bulgarian ground beetles from subfamilies Nebriinae, Carabinae and Cicindelinae (Coleoptera: Carabidae)
}

\author{
Teodora Marius Teofilova
}

Institute of Biodiversity and Ecosystem Research (IBER), Bulgarian Academy of Sciences (BAS), 1 Tsar Osvoboditel Blvd, 1000 Sofia, Bulgaria, oberon_zoo@abv.bg Z; https://orcid.org/0000-0003-0111-1573 [ك

\begin{abstract}
Bulgarian ground beetle (Coleoptera: Carabidae) fauna is relatively well studied but there are still many species and regions in the country which are not well researched. The present study aims at complementing the data about the distribution of the carabids from the subfamilies Nebriinae, Carabinae and Cicindelinae, which contain some of the most attractive Palaearctic carabids. Currently, 13 species of Nebriini, ten species of Notiophilini, one species of Cychrini, 28 species of Carabini and 15 species of Cicindelini are known to occur in Bulgaria. The paper gives new information and new records on 37 carabid species and 25 zoogeographical regions in Bulgaria. Beetles are collected in the period from 1976 to 2021 by different collectors and sampling methods. Six species are recorded for the first time in different regions. Four species are reported for the second time in the regions where they have currently been collected. Fourteen species haven't been reported for more than 20 years from the Middle and Western Stara Planina Mts, Western Bulgaria Region, Kraishte Region, Boboshevo-Simitli Valley, Vitosha Mts, Plana Mt, Lyulin Mts, Rila Mts and Pirin Mts.
\end{abstract}

Keywords: addition, Bulgaria, carabids, distribution

\section{Introduction}

Ground beetles (Coleoptera: Carabidae) represent one of the largest beetle families with cosmopolitan distribution and with decisive importance for the functioning of ecosystems. The representatives of the studied tribes Nebriini, Notiophilini, Cychrini, Carabini and Cicindelini are among the most attractive Palaearctic carabids, and often occur in many collections. In Bulgaria, most of these beetles are montane and forest dwellers or intra- and extrazonal ecotone and riparian species (e.g. Kryzhanovskij, 1983). They are mostly stenotopic or polytopic, nonflying zoophages, adapted to more mesophilic conditions, which is limiting their mass spreading and survival in anthropogenically modified ecosystems (e.g. Kryzhanovskij, 1983). Probably, due to that fact, there are still many regions and taxa continuing to surprise us with new records.

Ground beetles are relatively well studied in Bulgaria, but many regions are still poorly researched, and there are still many gaps in our knowledge, especially about the endemic taxa. According to the last edition of the Palaearctic Catalogue of the Ground Beetles, in Bulgaria currently 12 species of Nebriini, ten species of Notiophilini, one species of Cychrini, 24 species of Carabini and 16 species of Cicindelini are known (Löbl \& Löbl, 2017). However, according to our own recent estimations (Teofilova \& Guéorguiev, unpublished results) these numbers are not accurate, because Cicindela (Sophiodela) virgula Fleutiaux, 1894 never occurred in Bulgaria, but is included in the Palaearctic catalogue (probably by a technical mistake). Furthermore, Leistus (Leistus) piceus Frölich, 1799, Carabus (Carabus) menetriesi Faldermann in Hummel, 1827, C. (Archicarabus) nemoralis O. F. Müller, 1836, C. (Pachystus) hungaricus Fabricius, 1792, and C. (Oreocarabus) linnei Panzer, 1810 are missing for Bulgaria in the Palaearctic catalogue, but they are proved in the country (Guéorguiev \& Guéorguiev, 1995a; Turin et al. 2003; Teofilova et al. 2020; Teofilova \& Guéorguiev, unpublished results). Therefore, the total number of the species currently known in Bulgaria should be 13 
species of Nebriini, ten species of Notiophilini, one species of Cychrini, 28 species of Carabini and 15 species of Cicindelini.

The present study is the last part from the complex work concerning the unpublished data about Bulgarian carabids (Teofilova, 2021a, 2021b, 2021c, 2021d, 2021e). It aims at complementing the data about the distribution of the ground beetles from the subfamilies Nebriinae, Carabinae and Cicindelinae in Bulgaria by adding new records for $5 \%$ of the 747 species in Bulgarian carabid fauna (Teofilova \& Guéorguiev, unpublished results), collected in 25 zoogeographical regions and subregions (using the same zoogeographical division as in the last catalogue of Bulgarian carabids (Guéorguiev \& Guéorguiev, 1995a).

\section{Material and methods}

The majority of the material for this study came from a field work carried out in different localities in Bulgaria in the period from 1976 to 2021 . This material was collected through different sampling methods, such as handpicking, pan, Malaise, or pitfall trapping, and has not been published so far. All specimens are stored in the author's collection in the Institute of Biodiversity and Ecosystem Research (BAS, Sofia).

The following abbreviations of the collectors' names are used in the text: AV - A. Valkov, DC Dragan Chobanov, DS - Desislava Stoyanova, EM - E. Markova, GH - Georgi Hristov, IG - Iliya Gyonov, IT - Ivaylo Todorov, IV - Ilko Vassilev, KD - Krasimir Donchev, MN - Maria Naumova, MP - Magdalena Popova, NKar - Nikolay Karaivanov, NK - Nikolay Kodzhabashev, NS - Nikolay Simov, OI - Ognyan Iliev, OT - Ognyan Todorov, PBe - Petar Beron, PB P. Blagoev, RT - R. Todorov, SL - Simeon Lukanov, SSt - Stilian Stefanov, TL - Toshko Ljubomirov, TT Teodora Teofilova, YG - Yuli Ganev, VG - Vasil Genchev, VGu - Vyara Gusseva, VP - Vlada Peneva (Radkova), VS - Vladimir Sakalian.

The abbreviations of the zoogeographical regions and subregions (eastern, western or middle part) are as follows: BS - Black Sea Coast, DE - Eastern Danubian Plain, PRM - Middle Pre-Balkan (Predbalkan), PRW Western Pre-Balkan (Predbalkan), SPE - Eastern Balkan (Stara Planina) Mts, SPM - Middle Balkan (Stara Planina) Mts, SPW - Western Balkan (Stara Planina) Mts, SGS - Sashtinska Sredna Gora Mts, SGI - Ihtimanska Sredna Gora Mts, WB - Western Bul- garia, K - Kraishte Region, OP - Osogovska Planina Mt, MPO - Maleshevska Planina Mt and Ograzhden Mt, BSM - Boboshevo-Simitli Valley, SPT Sandanski-Petrich Valley, L - Lyulin Mts, V - Vitosha Mts, PL - Plana Mt, R - Rila Mts, P - Pirin Mts, T Thracian Lowland, ST - Sakar-Tundzha Region, RDW - Western Rhodope Mts, RDE - Eastern Rhodope Mts, S - Strandzha Mts.

The material was determined by the author unless otherwise noted in the text. All specimens determined by others were re-examined by the author. The nomenclature follows the last edition of the Catalogue of the Palaearctic ground beetles (Löbl \& Löbl, 2017).

\section{Results}

The present study is based on various field observations and contains data about 37 Bulgarian carabid species from tribes Nebriini (7), Notiophilini (6), Cychrini (1), Carabini (19) and Cicindelini (4), collected in 25 zoogeographical regions and subregions in Bulgaria. There are six species recorded for the first time in different subregions. Four species are reported for the second time in the regions where they were currently collected. Fourteen species haven't been reported for the given regions for more than 20 years.

Species list

Nebriinae Laporte, 1834

Nebriini Laporte, 1834

1. Leistus (Leistus) ferrugineus (Linnaeus, 1758). Material examined: V ("Vitocha", 1 +, no date, leg. VGu, det. O. Kryzhanovskij).

2. Leistus (Pogonophorus) magnicollis Motschulsky, 1866. Material examined: RDW (SE Pavelsko Vill., 41 ${ }^{\circ} 50^{\prime} 52^{\prime \prime} \mathrm{N}, 24^{\circ} 43^{\prime} 08^{\prime \prime} \mathrm{E}, 1100 \mathrm{~m}$, forest ecotone, 1 今ึ, 17.V.2016, leg. TT).

3. Leistus (Pogonophorus) rufomarginatus (Duftschmid, 1812). Material examined: V ("Vitocha", 1 Jै, 19.IX.1980, leg. IV, det. O. Kryzhanovskij).

4. Leistus (Pogonophorus) spinibarbis rufipes Chaudoir, 1843. Material examined: SPM (Leshnitsa Reserve, NW Yasenovo Vill., 4242'22"N, 2513'14"E, $658 \mathrm{~m}$, beech forest, 1 o, 1 +, 9.V-3.IX.2014, leg. TT); V ("Vitocha", 1 ô, 19.IX.1980, leg. IV, det. O. 
Kryzhanovskij; Bay Krastyo Site, about 1400 m, 1 ô, IX.1979, 1 q, 13.V.1980, leg./det. VP).

5. Nebria (Boreonebria) gyllenhali gyllenhali Schönherr, 1806. Material examined: R (Parangalitsa

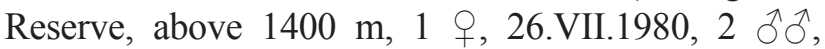
23.V.1982, leg. IV, det. A. Dostal; Parangalitsa Reserve, 1430 m, near Bistritsa River, 1 ô, 30.IV.1981, leg./det. VP).

6. Nebria (Eunebria) jockischii jockischii Sturm, 1815. Material examined: V ("Vitoscha", $1600 \mathrm{~m}, 1$ q, 27.VII.1983, 1500 m, 1 §ิ, 6.VIII.1983, leg. VGu, det. O. Kryzhanovskij).

7. Nebria (Nebria) brevicollis (Fabricius, 1792). Material examined: BS (Irakli Site, near Vaya River, $42^{\circ} 45^{\prime} 14 " \mathrm{~N}, 27^{\circ} 52^{\prime} 50^{\prime \prime} \mathrm{E}, 10 \mathrm{~m}, 1$ ex., 11.V.2021, leg. MN); PRW (Reselets Vill., 1 ô, 20.VI.1981, leg. IV, det. unknown); SGI (SE German Vill., 42 $36^{\prime} 03^{\prime \prime} \mathrm{N}$, $23^{\circ} 26^{\prime} 34^{\prime} \mathrm{E}, 790$ m, 1 9, 2.VI.1999, leg./det. NK); K (Razhdavitsa Vill., about $500 \mathrm{~m}$, meadow, 2 กิ $\widehat{\text {, }}$ X.1984, leg. VS); V (Knyazhevska Gora Park, about 800 m, 1 O, 16.IV.1980, leg./det. VP); RDW (Dobra

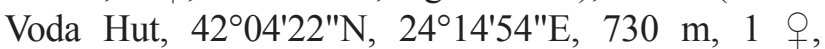
10.VII.1983, leg. PB, 1 ㅇ, leg. EM, det. unknown).

Notiophilini Motschulsky, 1850

8. Notiophilus aestuans Dejean, 1826. Material examined: BSM (Selishte Vill., 1 ex., 14.IX.1978, leg. VP, det. O. Kryzhanovskij).

9. Notiophilus biguttatus (Fabricius, 1779). Material examined: PRW (Reselets Vill., 1 \%, 20.VI.1981, leg. IV, det. A. Dostal); SPM (near Mazalat Hut, $42^{\circ} 45^{\prime} 44^{\prime \prime} \mathrm{N}, 25^{\circ} 08^{\prime} 03^{\prime \prime} \mathrm{E}, 1412 \mathrm{~m}, 1$ ex., 6.IX.2012, leg. TT\&NK); V ("Vitoscha", 1200 m, 1 , 28.IX.1985, leg. VGu, det. O. Kryzhanovskij).

10. Notiophilus laticollis Chaudoir, 1850. Material

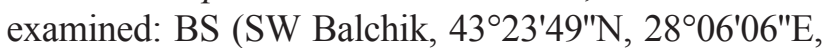
$218 \mathrm{~m}, 1$ ㅇ, 17.IV-10.V.2018, pitfall traps, leg. TL); MPO (Ograzhden Mts, 1 ô, 20.IV.1982, leg. YG, det. A. Dostal).

11. Notiophilus palustris (Duftschmid, 1812). Material examined: V ("Vitoscha", $2000 \mathrm{~m}, 1$ O, 4.VIII.1983, leg. VGu, det. O. Kryzhanovskij); R (Slavovo Vill., 1150 m, 1 , 31.X.1981, leg. VP, det. NK).

12. Notiophilus rufipes Curtis, 1829. Material examined: SPM (near the town of Shipka, about $700 \mathrm{~m}, 1$ ふૈ, 2 우, 25.V.1998, leg. AV); K (Razhdavitsa Vill., about $500 \mathrm{~m}, 1$ กे, 3.III.2001, leg. IG).
13. Notiophilus substriatus G.R. Waterhouse, 1833. Material examined: $\mathrm{T}$ (Yagodovo Vill., $42^{\circ} 06^{\prime} 38^{\prime \prime} \mathrm{N}, 24^{\circ} 51^{\prime} 04^{\prime \prime} \mathrm{E}, 159 \mathrm{~m}$, house yard, 1 \%, 11-20.VI.2020, leg. VG).

Carabinae Latreille, 1802

Cychrini Laporte, 1834

14. Cychrus semigranosus balcanicus Hopffgarten, 1881. Material examined: SPM (S Prestoy Vill., $42^{\circ} 50^{\prime} 20^{\prime \prime} \mathrm{N}, 25^{\circ} 30^{\prime} 26^{\prime \prime} \mathrm{E}, 709 \mathrm{~m}, 1$ \% , 1.VI.2012, leg.

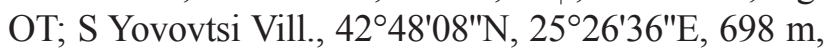
1 Oे, 6.IX.2012, leg. OT); L (Lyulin Mts, 1 क , 7.VII.1999, leg./det. NK).

Carabini Latreille, 1802

15. Calosoma (Calosoma) inquisitor inquisitor (Linnaeus, 1758). Material examined: SPT (Tisata Reserve, $41^{\circ} 43^{\prime} 31^{\prime \prime} \mathrm{N}, 2^{\circ} 08^{\prime} 53^{\prime \prime} \mathrm{E}, 230 \mathrm{~m}, 1$ ○े, 26.IV.2014, leg. TT); RDW (W Asenovgrad, $42^{\circ} 00^{\prime} 42^{\prime \prime} \mathrm{N}, 24^{\circ} 51^{\prime} 34 " \mathrm{~N}, 307 \mathrm{~m}, 2$ 우, 19.V.2002, leg. VG); S (S Kraynovo Vill., 4159'11"N, 2651'35"N, 480 m, 2 ○े ô, 30.VII.1989, leg. VG).

16. Calosoma (Calosoma) sycophanta sycophanta (Linnaeus, 1758). Material examined: RDE (Studen Kladenets Vill., 1 + , 18.V.1989, leg. RT).

17. Calosoma (Caminara) denticolle Gebler, 1833. Material examined: BS (Cape Maslen Nos, $42^{\circ} 18^{\prime} 27^{\prime \prime} \mathrm{N}, 27^{\circ} 47^{\prime} 34 " \mathrm{E}, 15 \mathrm{~m}$, at light, 1 , 24.VIII.1997, leg. NS).

18. Carabus (Archicarabus) montivagus montivagus Palliardi, 1825. Material examined: P (E Mozgovitsa Site, $41^{\circ} 40^{\prime} 50^{\prime \prime} \mathrm{N}, 23^{\circ} 24^{\prime} 40^{\prime \prime} \mathrm{E}, 1520 \mathrm{~m}, 1$ ex., 14.V.2014, leg. KD).

19. Carabus (Archicarabus) wiedemanni wiedemanni Ménétriés, 1836. Material examined: RDE (Kroyatsi Hut near Gurgulitsa Vill., 1 , 24.III.1990, leg./det. NK).

20. Carabus (Carabus) granulatus granulatus Linnaeus, 1758. Material examined: BS (Baltata Reserve near Balchik, 1 ô, 23.IV.1977, leg. unknown, det. O. Kryzhanovskij; N Krapets Vill., 4339'40"N, $28^{\circ} 33^{\prime} 58^{\prime \prime} \mathrm{E}, 1 \mathrm{~m}, 1$ q, 28.VIII.2020, leg. MN); DE (Srebarna Lake, 1 ô, 10.VII.1989, leg./det.NK).

21. Carabus (Chaetocarabus) intricatus intricatus Linnaeus, 1761. Material examined: SPM (S Prestoy 
Vill., 42 ${ }^{\circ} 50^{\prime} 20^{\prime \prime} \mathrm{N}, 25^{\circ} 30 ' 26 " \mathrm{E}, 709 \mathrm{~m}, 1$ ô, 1.VI.2012, leg. OT; N Shipka Peak, 4245'24"N, 25¹9'25"E, 1160 m, 1 9, 6.IX.2012, leg. OT); SPW (SW Zanozhene, Varshets, $43^{\circ} 09^{\prime} 05^{\prime \prime} \mathrm{N}, 23^{\circ} 13^{\prime} 51^{\prime \prime} \mathrm{E}, 780 \mathrm{~m}, 1$ ex., 30.IV.2014, leg. KD); SGI (SE German Vill., $42^{\circ} 36^{\prime} 03^{\prime \prime} \mathrm{N}, 23^{\circ} 26^{\prime} 34^{\prime \prime E}, 790$ m, 1 ô, 7.IX.1999, leg./ det. NK); L (Knyazhevo, 42³9'06"N, 2313'10"E, 706 $\mathrm{m}, 1$ o, 28.IV.2021, leg. SSt); V (Knyazhevo, $42^{\circ} 38^{\prime} 55^{\prime \prime N}, 23^{\circ} 13^{\prime} 09^{\prime \prime E}, 780 \mathrm{~m}, 1$ ex., 27.IV.2021, leg. SSt); PL (Planshtitsa River, 42 ${ }^{\circ} 33^{\prime} 15^{\prime \prime} \mathrm{N}, 2^{\circ} 27^{\prime} 89^{\prime \prime} \mathrm{E}$, 724 m, 1 ô, 24.V.2012, leg. MN); R (NW Dobarsko Vill., 415'ㄱ'13"N, 2325'51"E, 1600 m, 1 ô, 9.V.2020, leg. KD); RDW (SW Bachkovo Vill., 41 ${ }^{\circ} 56^{\prime} 40^{\prime \prime} \mathrm{N}$, $24^{\circ} 50^{\prime} 60^{\prime \prime} \mathrm{N}, 383 \mathrm{~m}, 1 \mathrm{o}^{\wedge}, 15 . \mathrm{VIII} .2003$, forest, under lamp, leg. VG; SE Markovo Vill., 42 $03^{\prime} 17^{\prime \prime N}$, 24ํํ'14"E, 456 m, 1 ex., 2.IX.2018, leg. KD).

22. Carabus (Eucarabus) ullrichi rhilensis Kraatz, 1876. Material examined: SPW (S Dalgi Del Vill., 4313'26"N, 2257'53"E, 940 m, 1 ex., 25.V.2014, leg. KD); WB (Sofia City, Borisova Gradina Park, $42^{\circ} 40^{\prime} 52^{\prime \prime N}, 2^{\circ} 20^{\prime} 32^{\prime \prime E}, 577 \mathrm{~m}, 1$ ㅇ, 5.IV.2016, 1 ㅇ, 18.V.2020, leg. TT); L (Knyazhevo, 42³9'06"N, 2313'10"E, $706 \mathrm{~m}, 1$ ô, 28.IV.2021, leg. SSt); V (Knyazhevo, 42 ${ }^{\circ} 38^{\prime} 55^{\prime \prime} \mathrm{N}, 23^{\circ} 13^{\prime} 09^{\prime \prime E}, 780$ m, 4 ex., 27.IV.2021, leg. SSt; Dragalevtsi, 1 ô, 24.VI.2021, leg. VP).

23. Carabus (Lamprostus) torosus torosus I. Frivaldszky von Frivald, 1835. Material examined: BS (SW Balchik, 432' $48^{\prime \prime} \mathrm{N}, 28^{\circ} 06^{\prime} 55^{\prime \prime} \mathrm{E}, 190 \mathrm{~m}, 1$ ㅇ,

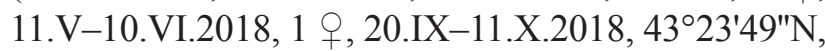

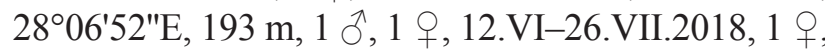

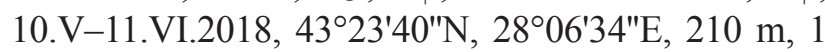

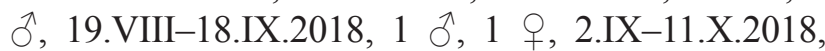
$43^{\circ} 23^{\prime} 31^{\prime \prime} \mathrm{N}, \quad 28^{\circ} 06^{\prime} 24^{\prime \prime} \mathrm{E}, \quad 214 \mathrm{~m}, 2$ 우, 10.V-11.VI.2018， 2 우， 12.VI-25.VII.2018, $43^{\circ} 23^{\prime} 49^{\prime \prime} \mathrm{N}, \quad 28^{\circ} 06^{\prime} 20^{\prime \prime} \mathrm{E}, 217 \mathrm{~m}, 1$ गे, 1 ㅇ, 17.IV-10.V.2018， 3 우， 19.VIII-18.IX.2018, $43^{\circ} 23^{\prime} 49^{\prime \prime} \mathrm{N}, \quad 28^{\circ} 06^{\prime} 06^{\prime \prime} \mathrm{E}, 218 \mathrm{~m}, 1 \mathrm{o}, 4$ 우, 10.V-11.VI.2018, 6 ふぇ,, 1 ㅇ, 12.VI-25.VII.2018, pit-

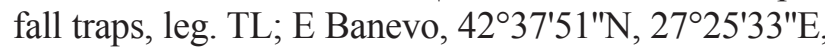
$168 \mathrm{~m}, 1$ ㅇ, 5.VI.2021, leg. DC).

24. Carabus (Megodontus) violaceus azurescens Dejean, 1826. Material examined: SPW (Petrohan Pass

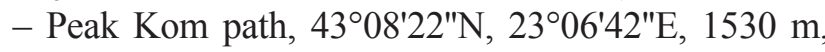
ridge meadow with juniper, 1 ex., 15.IV.2016, leg. TT); SGS (Koprivshtitsa, 42³8'25"N, 24²1'23"E, 1020 m, 1 ${ }^{2}$, 18.VIII.2016, leg. TT); SGI (SE German Vill.,

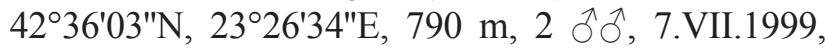
leg./det. NK); $\mathrm{R}$ (N Yakoruda, 42 $04^{\prime} 19 " \mathrm{~N}$, 233'ㄹ"E, $1629 \mathrm{~m}, 1$ ㅇ, 21.V.2015, leg. DS; S
Samokov, Mala Tsarkva Vill., 45¹5'14"N, 2330'44"E, 1203 m, 1 ô, 25-30.VI.2020, leg. IT); RDW (S

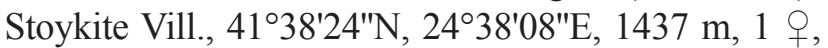
15.X.2020, leg. TT).

25. Carabus (Morphocarabus) praecellens versicolor I. Frivaldszky, 1835. Material examined: SPM (Gabrovo, 1 §̂, 20.V.1976, leg. unknown, det. NK).

26. Carabus (Pachystus) hortensis hortensis Linnaeus, 1758. Material examined: SGS (W Koprivshtitsa, Karavelova Polyana Site, $42^{\circ} 38^{\prime} 03^{\prime \prime N}$, $24^{\circ} 19^{\prime} 25^{\prime \prime} \mathrm{E}, 1274 \mathrm{~m}$, many beetles in an empty plastic bottle, 20.X.2013, leg. TT); V ("Vitocha", 1 Oे, 1989,

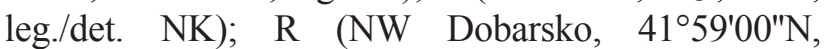
$23^{\circ} 26^{\prime} 20^{\prime \prime E}, 1520$ m, 1 ex., 25.VI.2019, leg. KD); P (SW Bansko, 41 466'38"N, 2326'02"E, 1655 m, 1 ex., 16.VIII.2014, leg. KD).

27. Carabus (Pachystus) cavernosus cavernosus I. Frivaldszky von Frivald, 1838. Material examined: OP (on the road to Ruen Peak, 42 ${ }^{\circ} 10^{\prime} 03^{\prime \prime} \mathrm{N}, 22^{\circ} 32^{\prime} 23^{\prime \prime} \mathrm{E}$, $2099 \mathrm{~m}$, open subalpine habitat, $1 \hat{\partial}, 17$. VI.2012, leg. TT).

28. Carabus (Pachystus) graecus morio Mannerheim, 1830. Material examined: BS (SW

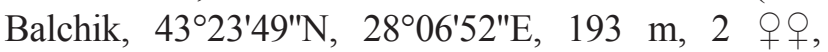

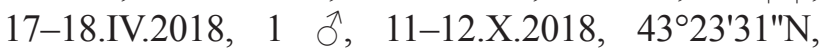

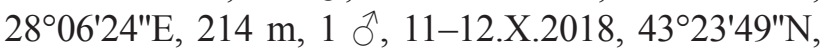
$28^{\circ} 06^{\prime} 06^{\prime \prime E}, 218 \mathrm{~m}, 1$ §, 11-12.X.2018, pan traps, leg.

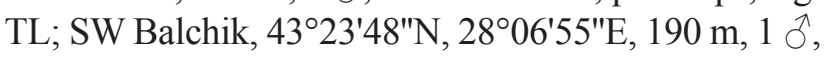

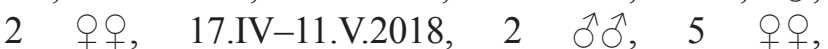
11.V-10.VI.2018, 432ㄱ' $49^{\prime \prime} \mathrm{N}, 28^{\circ} 06^{\prime} 52^{\prime \prime} \mathrm{E}, 193 \mathrm{~m}, 5$

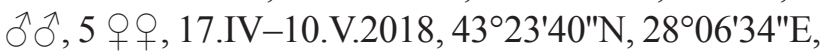

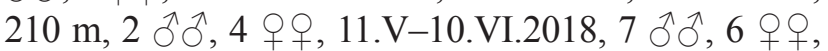
19.VIII-18.IX.2018, 1 ô, 9 우, 2.IX-11.X.2018,

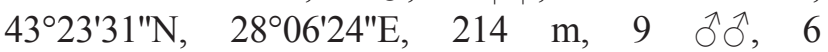
우,17.IV-10.V.2018, 1 ふึ, 3 우, 10.V-11.VI.2018, 1

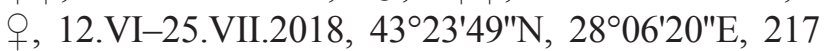

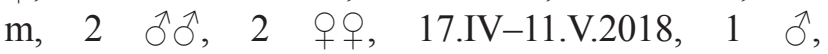
10.V-11.VI.2018, 2へ, 19.VIII-18.IX.2018, 8 ふぇ, 2

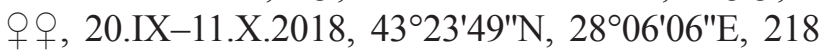
m, 4 ふ઼ิ, 2 우, 17.IV-10.V.2018, pitfall traps, leg. TL); SPT (Kozhuh Mt, about 230 m, 1 ڤึ, 20.X.1985, leg. YG, det. O. Kryzhanovskij); P (Tisata Reserve, $41^{\circ} 44^{\prime} 14^{\prime \prime} \mathrm{N}, \quad 23^{\circ} 10^{\prime} 37^{\prime \prime} \mathrm{E}, \quad 414 \mathrm{~m}, 4$ 우우, 25.IV-7.VI.2014, leg. TT); RDE (Studen Kladenets Vill., 1 ô, 18.V.1989, leg. RT).

29. Carabus (Procerus) gigas gigas Creutzer, 1799. Material examined: WB (W Bezden Vill., $42^{\circ} 52^{\prime} 53^{\prime \prime} \mathrm{N}, 23^{\circ} 05^{\prime} 55^{\prime \prime} \mathrm{E}, 561 \mathrm{~m}, 2$ ex., VI.2019, leg. NKar). 
30. Carabus (Procerus) scabrosus Olivier, 1790. Carabus (Procerus) scabrosus scabrosus Olivier, 1790. Material examined: T (Stara Zagora, Bedechka Park, 1 ex., 21.VI.2021, leg. MP); RDE (Studen Kladenets Vill., 1 ex., 18.V.1989, leg. RT; SW Rozino Vill., 41 ${ }^{\circ} 26^{\prime} 10^{\prime \prime} \mathrm{N}, 25^{\circ} 53^{\prime} 10^{\prime \prime} \mathrm{E}, 215 \mathrm{~m}$, mesoxerophilic oak forest near a tributary of the Byala Reka River, 1 ex., 12.V.2017, leg. KD). Carabus (Procerus) scabrosus bureschianus Breuning, 1928. Material examined: RDW (N Grashtitsa hamlet, 41 ${ }^{\circ} 39^{\prime} 16^{\prime \prime} \mathrm{N}$, 24³7'21"E, 1476 m, 1 ex., 6.V.2018, leg. TT; SE

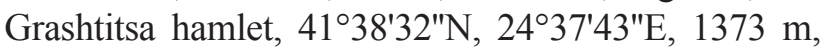
forest road, 2 larvae, 3.V.2019, leg. TT; Grashtitsa

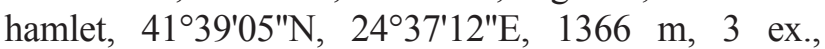
17-18.VII.2020, 41 $39^{\circ} 05^{\prime \prime} \mathrm{N}, 24^{\circ} 37^{\prime} 04^{\prime \prime} \mathrm{E}, 1340 \mathrm{~m}, 1$ larva, 14.X.2020, leg. TT).

31. Carabus (Procrustes) coriaceus Linnaeus, 1758. Carabus (Procrustes) coriaceus cerisyi Dejean, 1826. Material examined: SPM (N Shipka Peak, $42^{\circ} 45^{\prime} 24^{\prime \prime} \mathrm{N}, 25^{\circ} 19^{\prime} 25^{\prime \prime} \mathrm{E}, 1160 \mathrm{~m}, 1$ §ै, 6.IX.2012, leg. OT); WB (W Bezden Vill., 42 $52^{\prime} 54^{\prime \prime} \mathrm{N}, 2^{\circ} 05^{\prime} 48^{\prime \prime} \mathrm{E}$, $558 \mathrm{~m}, 3 \partial^{\lambda}, 1$ ㅇ, 5.IV.2018, leg. NKar); SPT (Tisata

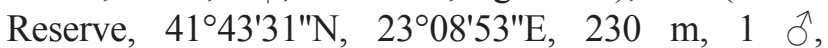
25.IV.2014, leg. TT); L (Lyulin Mts, 2 우, 7.VII.1999, leg./det. NK); R (S Samokov, Mala Tsarkva Vill.,

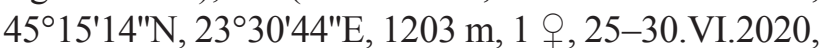

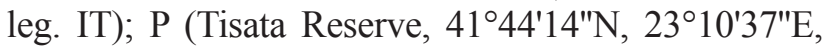
414 m, 2 ठิठ઼, 25.IV-7.VI.2014, leg. TT). Carabus (Procrustes) coriaceus kindermanni Waltl, 1838.

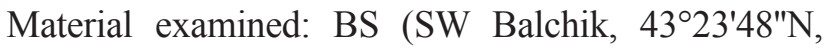
$28^{\circ} 06^{\prime} 55^{\prime \prime} \mathrm{E}, 190 \mathrm{~m}, 1$ क , 17.IV-11.V.2018, 1 ठै, 19.VIII-18.IX.2018, 1 ㅇ, 20.IX-11.X.2018, 1 ㅅ, 1 우,

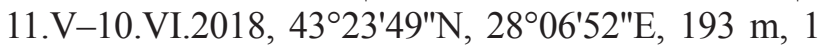

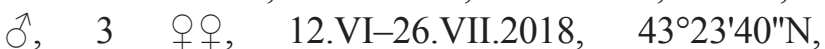
2806'34"E, 210 m, 1 ภ, 3 우, 19.VIII-18.IX.2018, 1

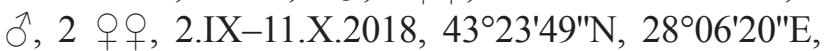
217 m, 2 ๙ึ่, 19.VIII-18.IX.2018, 2 우, 20.IX-11.X.2018, pitfall traps, leg. TL; NW Lozenets Vill., 42 ${ }^{\circ} 12^{\prime} 52^{\prime \prime} \mathrm{N}, 27^{\circ} 47^{\prime} 50^{\prime \prime} \mathrm{E}, 11 \mathrm{~m}, 1$ ㅇ, 23.V.2021, leg. KD); T (NW Kostievo Vill., $42^{\circ} 11^{\prime} 13^{\prime \prime} \mathrm{N}$, $24^{\circ} 37^{\prime} 02^{\prime \prime E}, 175 \mathrm{~m}, 1$ ㅇ, 27.IV.2018, leg. TL; S

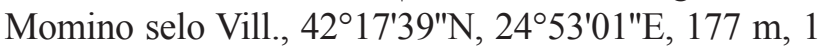
, 12.VI.2018, pasture, pan trap, leg. TL; W Elenino Vill., 42²1'45"N, 25³4'34"E, 200 m, 1 ex., 2.X.2019, leg. KD); ST (Branitsa-Yerusalimovo road, near Harmanli, 41 ${ }^{\circ} 56^{\prime} 12^{\prime \prime} \mathrm{N}, 26^{\circ} 05^{\prime} 46^{\prime \prime} \mathrm{E}, 207 \mathrm{~m}, 1$ ex., 4.XI.2010, leg. KD).

32. Carabus (Tomocarabus) convexus Fabricius, 1775. Carabus (Tomocarabus) convexus dilatatus Dejean, 1826. Material examined: SGI (SE German

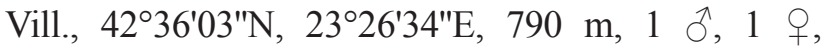

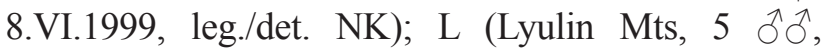
15.V.1999, leg./det. NK). Carabus (Tomocarabus) convexus gracilior Géhin, 1885. Material examined: PRM (NE Emen Vill., kanyon of Negovanka River, $43^{\circ} 08^{\prime} 35^{\prime \prime} \mathrm{N}, 25^{\circ} 22^{\prime} 20^{\prime \prime} \mathrm{E}$, about $190 \mathrm{~m}$, forest, 1 oै, 3.III.2001, leg. NS); SPT (Tisata Reserve, $41^{\circ} 45^{\prime} 42^{\prime \prime} \mathrm{N}$, $23^{\circ} 08^{\prime} 58^{\prime \prime} \mathrm{E}, 230 \mathrm{~m}, 1$ 今, 3 우, 25.IV-7.VI.2014, leg. TT); ST (Sakar Mt, Kostur Vill., 41 ${ }^{\circ} 58^{\prime} 22^{\prime \prime N}$, $26^{\circ} 17^{\prime} 10^{\prime \prime} \mathrm{N}, 455$ m, 1 ㅇ, 12.IV.2012, leg. VG); S (S Kraynovo Vill., 41 $59^{\prime} 11^{\prime \prime N}, 26^{\circ} 51^{\prime} 35^{\prime \prime} \mathrm{N}, 480$ m, 2

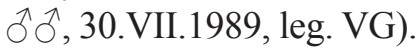

33. Carabus (Trachycarabus) scabriusculus bulgarus Lapouge, 1908. Material examined: WB (Chelopechene near Sofia, 1 + , 25.V.1993, leg. OI, det. NK).

Cicindelinae Latreille, 1802

Cicindelini Latreille, 1802

34. Cicindela (Cicindela) campestris Linnaeus, 1758. Cicindela (Cicindela) campestris campestris Linnaeus, 1758. Material examined: SPW (N Petrohan, $43^{\circ} 08^{\prime} 33^{\prime \prime} \mathrm{N}, 23^{\circ} 07^{\prime} 27^{\prime \prime} \mathrm{E}, 1400 \mathrm{~m}$, clearing, 1 \%, 15.IV.2016, leg. TT); OP (S Kyustendil, 42 ${ }^{\circ} 10^{\prime} 30^{\prime \prime} \mathrm{N}$, 2237'40"E, 1530 m, 1 ex., 16.VI.2012, leg. TT); L (Dragichevo Vill., about 750 m, 1 Oे, 30.VIII.1982, leg. IV, det. H. Freude; Knyazhevo, 42 $39^{\prime} 06^{\prime \prime N}$, $23^{\circ} 13^{\prime} 10^{\prime \prime E}, 706$ m, 1 Ô, 27.IV.2021, leg. SSt); R (Skakavitsa, 1 Jै, 17.VII.1983, leg. YG; Belmeken Dam, 4290'15"N, 2347'22"E, 1933 m, 1 , 26.IX.2014, leg. SL); P (Between Peak Vihren and Hut Vihren, 2400 m, 1 क, 30.VI.1971, leg. PBe, det. O. Kryzhanovskij; Dobrinishte, house yard, 13.IV.2021, leg. VP); RDW (N Grashtitsa hamlet, $1476 \mathrm{~m}$, $41^{\circ} 39^{\prime} 16^{\prime \prime N}, 24^{\circ} 37^{\prime 2} 21^{\prime \prime E}, 1$ ㅇ, 6.V.2018, leg. TT; Grashtitsa hamlet, 4138'34"N, 2437'32"E, 1350 m, 1 ex., 3.V.2019, leg. TT; NW Grashtitsa hamlet, $41^{\circ} 39^{\prime} 37^{\prime \prime} \mathrm{N}, 2^{\circ} 36^{\prime} 14^{\prime \prime E}, 1200 \mathrm{~m}, 1$ ex., 20.IV.2020, leg. TT). Cicindela (Cicindela) campestris palustris Motschulsky, 1840. Material examined: BS (SW Balchik, $43^{\circ} 23^{\prime} 49^{\prime \prime} \mathrm{N}, \quad 28^{\circ} 06^{\prime} 06^{\prime \prime} \mathrm{E}, 218 \mathrm{~m}, 1$ ठै, 17.IV-10.V.2018, leg. TL).

35. Cicindela (Cicindela) hybrida hybrida Linnaeus, 1758. Material examined: RDW (SE Turun Vill., $41^{\circ} 32^{\prime} 48^{\prime \prime} \mathrm{N}, 24^{\circ} 52^{\prime} 53^{\prime \prime} \mathrm{E}, 689 \mathrm{~m}, 2$ 우우, 6.VIII.2018, leg. TL).

36. Cicindela (Cicindela) sylvicola Dejean, 1822. Material examined: SPE (S Varbitsa Vill., Varbitsa 


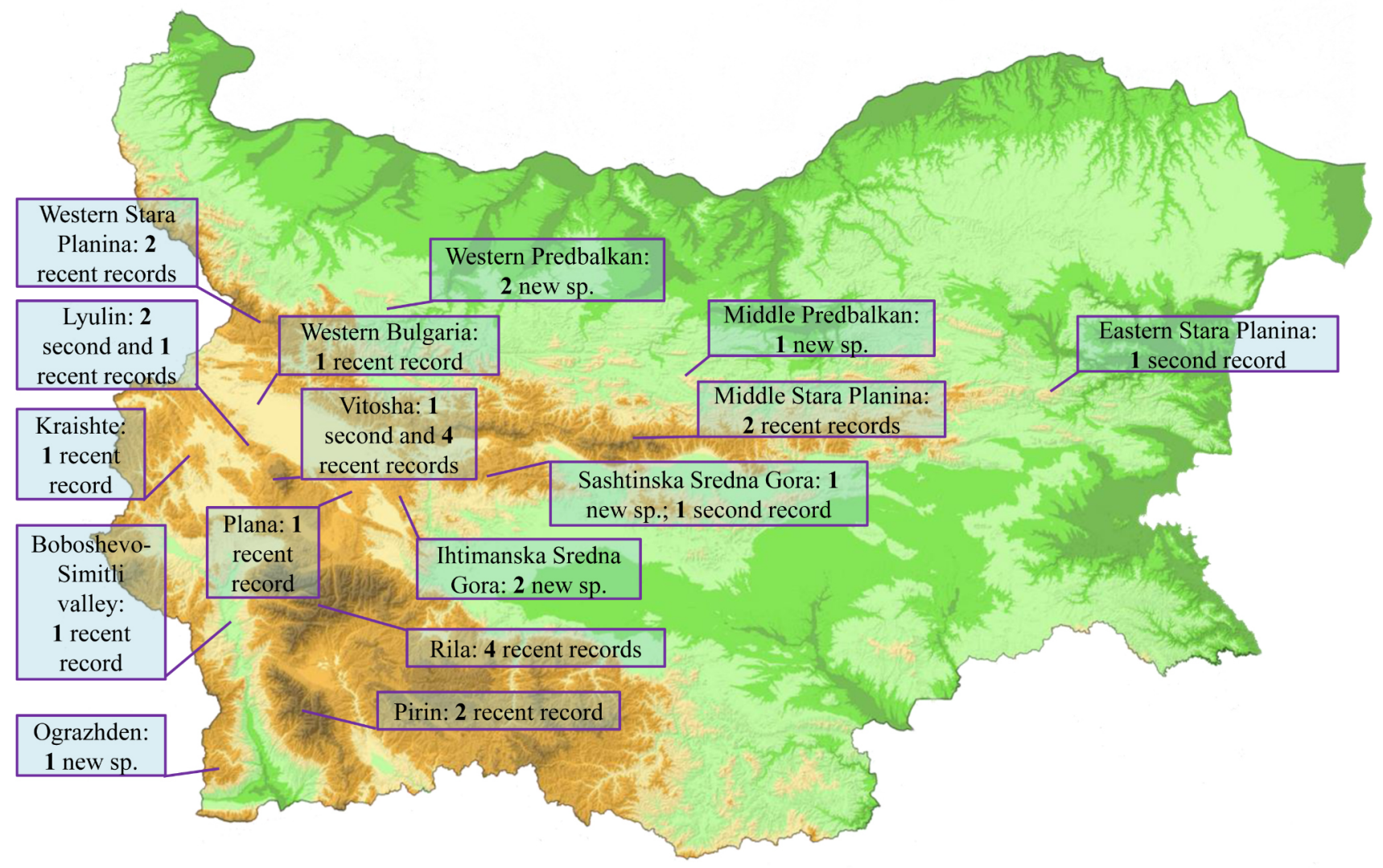

Fig. 1. Map of the regions where new data are recorded, with a respective number of the established species.

Pass, 2 웅, 20.V.2011, leg. TL); SPW (N Petrohan, $43^{\circ} 08^{\prime} 33^{\prime \prime} \mathrm{N}, 23^{\circ} 07^{\prime} 27^{\prime \prime} \mathrm{E}, 1400 \mathrm{~m}$, clearing, 2 ふึَ, 1 ㅇ, 13.VII.2017, leg. TT); SGI (NE Dolni Pasarel Vill., 4233'05"N, 2331'12"E, 967 m, 1 ô, 30.VI.2016, leg. $\mathrm{GH})$.

37. Cylindera (Cylindera) germanica germanica (Linnaeus, 1758). Material examined: L (Lyulin Mts, 1 १, 7.VII.1999, leg./det. NK).

\section{Discussion}

The present study provides some novelty about the carabid fauna of Bulgaria, in particular, of the tribes Nebriini, Notiophilini, Cychrini, Carabini and Cicindelini. They are well studied in Bulgaria, and have a great number of species of conservation value (endemic, relict, rare and two protected species). Despite that fact, this paper gives some new data on their distribution.

Six of the presented species are recorded for the first time in different regions in Bulgaria (Fig. 1):
Carabus convexus is new to the Middle Pre-Balkan, and Nebria brevicollis and Notiophilus biguttatus are new both to the Western Pre-Balkan and to the whole Pre-Balkan Region; Carabus violaceus is new to the Sashtinska Sredna Gora Mts; Nebria brevicollis and Cicindela sylvicola are new to the Ihtimanska Sredna Gora Mts; Notiophilus laticollis is reported here for the first time from the Ograzhden Mts.

Four species are reported for the second time in the regions where they were currently collected (Fig. 1). Cicindela sylvicola is now reported for the first time in Eastern Balkan Mts, since 1904 (Apfelbeck, 1904). Notiophilus palustris is reported only by Stoyanov and Penev (2004) for the Vitosha Mts. Cicindela campestris and Cylindera germanica haven't been reported for the Lyulin Mt, since Kodzhabashev \& Mollov (2000).

Fourteen species haven't been reported for more than 20 years from the Middle and Western Balkan Mts, Western Bulgaria, Kraishte Region, BoboshevoSimitli Valley, Vitosha Mts, Plana Mt, Lyulin Mts, Rila Mts and Pirin Mts (Fig. 1). Guéorguiev \& Guéorguiev 
(1995a) are the last who recorded: Leistus spinibarbis for the Middle Balkan Mts; Cicindela sylvicola for the Western Balkan Mts; Notiophilus rufipes for the Middle Balkan Mts and Kraishte Region; Carabus ullrichi for the Western Balkan Mts and Western Bulgaria Region; Notiophilus aestuans for the Boboshevo-Simitli Valley; Notiophilus biguttatus, Leistus ferrugineus and Nebria jockischii for the Vitosha Mts; Cicindela campestris and Notiophilus palustris for the Rila Mts; Carabus hortensis for the Rila Mts and Pirin Mts; Carabus intricatus for the Rila Mts and Plana Mt.; Carabus coriaceus for the Lyulin Mts. Same year, Notiophilus biguttatus and Leistus ferrugineus are also recorded for the last time in the Vitosha Mts by Guéorguiev \& Guéorguiev (1995b) and Krusteva et al. (1995), respectively. The last records of Carabus intricatus from the Vitosha Mts are by Guéorguiev \& Guéorguiev (1995a) and Krusteva et al. (1995). Carabus montivagus haven't been reported from the Pirin Mts since 1997 (Guéorguiev et al., 1997).

Some of these newly rediscovered species are quite common in Bulgaria and their long-time absence in the given regions is probably due to the lack of more recent research (e.g. Cicindela campestris, Carabus coriaceus and Notiophilus rufipes), since many areas in the country are missing contemporary information about their carabid (or any) fauna. Other species are relatively rare or stenotopic and do not fall in mass collections (e.g. Carabus montivagus, Leistus spinibarbis and Nebria jockischii).

During the research some interesting or confirmative data were obtained. Rare stenotopic forest mesophile Leistus magnicollis was found in the forest ecotone near sparsely vegetated stony habitat; included in the Red Data Book of the Republic of Bulgaria as vulnerable (Guéorguiev, 2015) Carabus scabrosus was also established in some ecotone habitats; mesophilous nature of Leistus spinibarbis and Carabus hortensis was confirmed, as they were found in beech forests; stenotopic hygrophilous mountain species Nebria gyllenhali and $N$. jockischii were collected near river on $1430 \mathrm{~m}$ a.s.1., and on 1500-1600 m, respectively; conservationally significant forest mesophile and indicator Carabus intricatus (IUCN 2021 - NT) was collected in different typically montane localities; rare steppe species Carabus torosus was found on places where it is known it occurs; rare Carabus cavernosus was observed in open subalpine habitat; relict montane species Nebria gyllenhali and Carabus hortensis were collected in their typical places of habitation. Cylindera germanica is widespread but rare in Bulgaria, thus every record of the species is valuable.

Although the ground beetle fauna of Bulgaria is relatively well studied, there are still some regions representing "white spots" in relation to the knowledge about their carabid species composition and community structure (e.g. many different parts of the Danubian Plain and Balkan Mts, Pre-Balkan Region and Sub-Balkan Valleys, Sakar Mts, some mountains near Sofia, Mesta River Valley), and these areas deserve some enlightening in relation to their faunas. More complete knowledge of the diversity of the studied group in the country could be revealed only after carrying out further research in the poorly-examined regions.

\section{Acknowledgments}

The author is grateful to all collectors for providing specimens. This paper is part of the ANIDIV Project of the IBER - BAS.

\section{References}

Apfelbeck V. 1904 Die Käferfauna der Balkanhalinsel, mit Berücksichtigung Klein-Asiens und der Insel Kreta. Erstes Band: Familienreihe Caraboidea. R. Friedlander und Sohn, Berlin, 422 pp.

Guéorguiev B.V. 2015 Annex 1.2 List of the invertebrate species assessed as "endangered" and "vulnerable": Coleoptera: Carabidae. In: Golemanski V., Stoev P., Dobrev D., Beron P., Zhivkov M., Popov A., Popov V., Beschkov V., Deltshev Ch., Michev T., Spassov N. (eds) Red Data Book of the Republic of Bulgaria. Volume 2, Animals, Sofia, BAS et MoEW, 354-355.

Guéorguiev V.B., Guéorguiev B.V. 1995a Catalogue of the ground-beetles of Bulgaria (Coleoptera: Carabidae). Pensoft Publishers, Sofia-Moscow, 279 pp.

Guéorguiev V.B., Guéorguiev B.V. 1995b La faune des Carabidae (Coleoptera) des hautes montagnes de Bulgarie. Acta zoologica bulgarica 48: 77-85.

Guéorguiev V.B., Sakalian V.P., Guéorguiev B.V. 1997 Biogeography of the endemic Balkan groundbeetles (Coleoptera: Carabidae) in Bulgaria. Pensoft Publishers, Sofia-Moscow, 73 pp. 
Hieke F., Wrase D.W. 1988 Faunistik der Laufkäfer Bulgariens (Coleoptera, Carabidae). Deutsche Entomologische Zeitschrift, N. F. 35 (1-3): 1-171. https://doi.org/10.1002/mmnd.19880350102 [

IUCN 2021 The IUCN Red List of Threatened Species. Version 2021-2. https://www.iucnredlist.org [ (accessed 28 November 2021)

Kodzhabashev N.D., Mollov K.B. 2000 Faunistic and ecological studies on the carabid fauna of the open territories in the vicinity of city of Sofia. Jubilee Book of Scientific Papers, Forestry University, Sofia, pp. 291-302. (In Bulgarian)

Krusteva I., Popov V., Sakalian V. 1995 Indirect gradient analysis of carabid (Coleoptera, Carabidae) spatial pattern on Vitosha Mountain (West Bulgaria). Third National Scientific Conference on Entomology, 18-20 September 1995, Sofia, pp. $55-59$.

Kryzhanovskij O.L. 1983 Fauna of the USSR. Coleoptera, Vol. 1, Is. 2. The ground-beetles of suborder Adephaga: families Rhysodidae, Trachypachidae; family Carabidae (Introduction, overview of the fauna of the USSR). Nauka, Leningrad, 341 pp. (In Russian)

Löbl I., Löbl D. (eds) 2017 Catalogue of Palaearctic Coleoptera. Archostemata-Myxophaga-Adephaga. Vol. 1. Revised and updated edition. Koninklijke Brill NV, Leiden, 1443 pp.

Stoyanov I., Penev L. 2004 Spatial structure of carabid beetle assemblages along an urban-rural gradient. In: Penev L., Niemelä J., Kotze D.J., Chipev N. (eds) Ecology of the City of Sofia. Species and Communities in an Urban Environment, pp. 371-400.
Teofilova T. 2021a New and unpublished data about Bulgarian ground beetles of the tribe Lebiini (Coleoptera: Carabidae). ZooNotes 174: 1-4.

Teofilova T. 2021b New and unpublished data about the ground beetles (Coleoptera: Carabidae) of Bulgaria. ZooNotes 175: 1-4.

Teofilova T. 2021c New and unpublished data about Bulgarian ground beetles from the tribes Pterostichini, Sphodrini and Platynini (Coleoptera: Carabidae). Acta biologica sibirica 7: 125-141. https://doi.org/10.3897/abs.7.e67015 [

Teofilova T. M. 2021d New and unpublished data about the ground beetles (Coleoptera: Carabidae) from the subfamily Trechinae of Bulgaria. Journal of insect biodiversity 26 (2): 39-45. https://doi.org/10.12976/jib/2021.26.2.3 [

Teofilova T. 2021e New and unpublished data about the ground beetles from tribes Zabrini and Harpalini (Coleoptera: Carabidae) of Bulgaria. Biharean biologist 15 (2): 131-138.

Teofilova T.M., Guéorguiev B.V., Kodzhabashev N.D. 2020 New and interesting data on the ground beetles (Coleoptera: Carabidae) from Bulgaria. Acta zoologica bulgarica 72 (2): 225-233.

Turin H., Penev L., Casale A. (eds) 2003 The Genus Carabus in Europe. A. Synthesis. Co-published by Pensoft Publishers, Sofia-Moscow et European Invertebrate Survey, Leiden, xvi +512 pp. 